\title{
La « phrase » en français médiéval : une réalité ou une reconstruction artificielle?
}

\author{
Alexei Lavrentiev \\ UMR ICAR - CNRS, Université de Lyon \\ Alexei.Lavrentev@ens-lyon.fr
}

\section{Introduction}

La notion de "phrase » joue un rôle important, sinon central, dans la syntaxe selon les grammaires traditionnelles. C'est la plus grande unité d'analyse grammaticale. Des théories syntaxiques plus récentes - générativistes (Chomsky 1986), structurelles (Tesnière 1965), dépendancielles (Mel'čuk 1988), etc. - recourent également à la notion de «nœud central » ou d'« arbre » à l'intérieur duquel on analyse des relations syntaxiques. Même si on sait grâce à la grammaire textuelle que des rapports grammaticaux (et notamment des relations anaphoriques) peuvent dépasser les limites de phrases, il semble évident qu'il existe une frontière au-delà de laquelle il n'est plus question de dépendance ni de règles combinatoires purement syntaxiques.

Et pourtant cette notion d' « unité maximale » reste non définie ou sa définition manque de précision. Ch. Marchello-Nizia (1979 : 42) a relevé, dans les grammaires scolaires essentiellement, au moins six critères donnés soit comme absolus, soit comme complémentaires, dont en effet « aucun n'est un critère absolu de reconnaissance ». Sont ainsi cités le critère graphique (la présence d'une ponctuation « forte »), le critère prosodique (la présence d'une pause et d'une intonation particulière), le critère sémantique (la présence d'un «sens complet »), le critère logique (la présence du thème et du propos), ainsi que deux critères proprement grammaticaux (autonomie et une certaine organisation formelle). M. Riegel et al. (1997) rejettent également les critères graphique, phonétique et sémantique et penchent pour une définition « structurelle ».

Sans tenter pour l'instant de trancher parmi les différentes définitions de phrase possibles, notons que la pertinence de cette notion par rapport aux textes en français médiéval a été mise en doute par plusieurs linguistes. Ainsi, C. Buridant n'y recourt pas dans sa Grammaire nouvelle de l'ancien français (2007). $\mathrm{Ch}$. Marchello-Nizia précise dans une note de son étude pionnière de la ponctuation française médiévale dans un cadre linguistique :

\begin{abstract}
J'utilise le mot "proposition" plutôt que "phrase simple" ou "phrase minimale" qui, en linguistique, désigne proprement une unité "abstraite", et non une portion d'énoncé découpée empiriquement, comme c'est le cas ici ; ces propositions que je repère d'après les critères de la grammaire traditionnelle (présence d'un verbe conjugué), correspondent à des "phrases minimales" sans doute, soit autonomes, soit reliées les unes aux autres, soit enchâssées les unes dans les autres ; mais aussi longtemps qu'on n'a pas élaboré une grammaire générative de ces états anciens de la langue, il semble prudent d'éviter d'employer un terme qui suppose cette élaboration achevée (Marchello-Nizia $1978: 36$ ).
\end{abstract}

La grille de classement d'« unités ponctuables » proposée par A. Lavrentiev (2007a ; cf. 2009) pour l'étiquetage d'un corpus de transcriptions de manuscrits médiévaux passe directement des unités de macrostructure textuelle aux frontières entre propositions.

B. Combettes ne situe qu'au XVI ${ }^{\mathrm{e}}$ siècle l'émergence de cette unité dans l'usage et dans la pensée linguistique (2000:63), et ce n'est guère qu'au XVII ${ }^{\mathrm{e}}$ s. que la notion moderne de «phrase » (qui avait une signification différente auparavant) est introduite dans les grammaires. Une observation semblable est faite par G. Nunberg au sujet de textes anglais : une «phrase textuelle» ne se forme en tant que catégorie grammaticale que sous l'influence de l'imprimerie, sans doute pas avant le XVII' siècle : 
Malgré les réserves exprimées par les linguistes, les éditeurs scientifiques des textes médiévaux n'hésitent pas, en «modernisant» la ponctuation des œuvres qu'ils publient, à y introduire des points et d'autres ponctuations fortes qui dès lors imposent, sans doute anachroniquement, une certaine segmentation phrastique.

Il convient de noter que malgré toutes ses insuffisances le critère graphique (la ponctuation forte) reste le plus opérationnel pour les outils de traitement automatique de la langue naturelle. A son tour, la segmentation en "phrases» peut avoir une incidence importante sur le fonctionnement des outils d'étiquetage morphologique et de « parsage» syntaxique automatique. Par ailleurs, dans la recherche des cooccurrences, la prise en compte des limites de phrases peut s'avérer extrêmement utile pour limiter le « bruit » éventuel.

Les «phrases » introduites par les éditeurs sont bien présentes dans les corpus de français médiéval fondés sur des éditions modernes. Elles peuvent avoir une incidence sur les résultats de l'interrogation de ces corpus, malgré l'absence de définition linguistique précise de cette notion et les doutes concernant sa pertinence pour la langue de l'époque. Dans la suite de cet article, nous tenterons de proposer des critères linguistiques précis, indépendants de la ponctuation, permettant un découpage en « unités syntaxiques maximales » de textes en français médiéval et nous confronterons ces critères théoriques à la pratique de la ponctuation des scribes médiévaux d'une vingtaine de manuscrits en prose, ainsi qu'à la ponctuation des éditions modernes de ces mêmes textes.

Les réflexions présentées ci-dessous sont inspirées par notre travail de thèse sur la ponctuation médiévale (Lavrentiev 2009) et par les discussions auxquelles nous avons participé dans le cadre du projet de constitution d'un corpus syntaxique de français médiéval SRCMF ${ }^{1}$.

\section{2 "Phrase graphique » et " unité syntaxique maximale »}

D. Creissels (1995 : $31 ; c f$. 2006) remarque que le terme de phrase est en effet couramment utilisé pour recouvrir deux notions qu'il convient de bien distinguer. D'une part, ce terme « renvoie à la possibilité de segmenter un texte en fragments successifs, segmentation qui se concrétise à l'écrit par l'usage du point ». Les tentatives des grammaires traditionnelles de mettre cette notion en rapport direct avec les pauses de l'oral et avec l'autonomie sémantique et d'en faire ainsi une notion de base de syntaxe lui paraissent vouées à l'échec. En revanche, il existe, selon le chercheur, une capacité universelle des signes linguistiques de former des combinaisons qui se caractérisent par une certaine «complétude syntaxique » :

Les locuteurs d'une langue ont l'intuition de différence de nature entre les énoncés qu'ils utilisent effectivement dans les conditions réelles de l'activité langagière. Certains énoncés sont reconnus comme «complets» lorsqu'on les soumet au jugement des locuteurs en dehors même de leurs conditions réelles d'utilisation, alors que d'autres, bien qu'attestées au moins dans certaines conditions d'énonciation, ne sont pas (ou ne sont que difficilement) acceptés comme tels... On peut faire l'hypothèse que les énoncés... « syntaxiquement achevés » ont comme caractéristique essentielle de permettre d'observer dans leur plénitude les lois combinatoires (Creissels $1995: 32-33)$.

A partir de cette intuition de « complétude syntaxique » observée chez les personnes « lettrées » aussi bien que chez les locuteurs de langues à tradition orale n'ayant reçu aucune éducation grammaticale, et en procédant à une série de tests sur les possibilités de modalisation énonciative, le linguiste arrive à la définition de l'«unité phrastique» qui «repose sur la possibilité d'analyser deux énoncés ou deux fragments d'énoncés comme représentant la même conceptualisation d'un événement à travers deux élaborations énonciatives différentes » (Creissels 1995 : 35). Cette deuxième notion est donc universelle et pourrait constituer le noyau de toute analyse syntaxique. 
Si on adopte ce point de vue qui distingue la "phrase» au sens traditionnel (que nous appellerons phrase graphique) de l'« unité phrastique» (que nous appellerons unité syntaxique maximale pour éviter toute ambiguïté), la problématique du découpage en «phrases » de textes en français médiéval se présente sous un nouvel angle. On pourrait ainsi découper les textes d'un corpus de travail en unités syntaxiques maximales et analyser ensuite leurs rapports avec les phrases graphiques des sources primaires et des éditions modernes.

Si la définition de la phrase graphique dans des éditions modernes ne pose pas de problème majeur (c'est une séquence textuelle délimitée par des marques de ponctuation fortes, telles que le point, le point d'exclamation ou d'interrogation ou encore le saut de ligne dans la majorité de leurs emplois), certaines précisions sont nécessaires quant à son application aux sources primaires médiévales. La notion d'unité syntaxique maximale doit également être précisée, compte-tenu de l'absence de locuteurs « natifs » de la langue médiévale et de l'impossibilité conséquente de procéder à des tests de l'intuition de « complétude syntaxique » et des possibilités de transformation.

\section{1 «Unités de lecture » dans les manuscrits médiévaux}

Grâce aux recherches menées depuis une trentaine d'années et notamment aux travaux de Ch. MarchelloNizia (1978 ; 2007), C. Buridant (1980), C. Barbance-Guillot (1995), E. Llamas Pombo (2001; 2007 ; 2008), S. Baddeley (2001), A. Lavrentiev (2000; 2007a ; 2007b ; 2007c ; 2009), C. Pignatelli (2007) et N. Mazziotta $(2008 ; 2009)$ consacrés à la ponctuation française médiévale, ainsi qu'aux ouvrages fondamentaux de M. Parkes (1992) et B. Mortara Garavelli (éd. 2008) qui traitent de l'histoire de la ponctuation dans toute l'Europe, nous disposons désormais d'une quantité suffisante de données pour dresser un inventaire des principales marques de ponctuation médiévale et pour comprendre les grandes tendances de leur fonctionnement.

Si l'éventail des marques de ponctuation utilisées dans la plupart des manuscrits français médiévaux est relativement stable, la fréquence de leur emploi varie de moins de deux à plus de quinze marques pour cent mots (Lavrentiev 2007a). La grande variabilité des pratiques de la ponctuation a été remarquée par tous les spécialistes qui ont mené des études comparatives de plusieurs manuscrits médiévaux :

\footnotetext{
Ce qui se révèle fondamentalement dans l'étude de la ponctuation, c'est la singularité de chaque manuscrit. Même lorsque deux ou plusieurs manuscrits offrent des versions presque identiques [...] du même " texte ", jamais la ponctuation n'est la même, et elle diffère toujours largement d'une leçon à l'autre, d'un manuscrit à l'autre (Marchello-Nizia 1978 : 42);

Chaque scribe établit son propre sous-système, sa propre distribution de valeurs ; $[\ldots]$ cela se traduit en une large polyvalence des signes ; ... le scribe n'applique pas toujours à l'intérieur d'un même codex les critères suivis dans une section déterminée (Llamas Pombo 2001, 153);

Ces manuscrits ... présentent une ponctuation assez riche... et relativement régulière à l'intérieur d'un texte donné, même si cette ponctuation diffère d'un texte à l'autre (Baddeley, 2001, 139).
}

Malgré cette diversité apparente, la grande majorité des occurrences de la ponctuation dans les manuscrits médiévaux se situe à ce que l'on peut appeler des «frontières ponctuables" (Lavrentiev 2007a) ou des « lieux de ponctuation » (Pignatelli 2007) : des endroits dans la chaine écrite où un ou plusieurs facteurs syntaxiques, sémantiques ou pragmatiques favorisent l'usage de la ponctuation. Si cet usage n'est jamais entièrement systématique, il est tout-à-fait possible de décrire les conditions syntaxiques dans lesquelles les chances de rencontrer une marque de ponctuation sont les plus élevées.

Le terme de phrase graphique est sans doute inapproprié aux données de manuscrits médiévaux, comptetenu de leur variabilité et du fait que la différence entre l'unité qui se dégage de ces données et la notion de phrase traditionnelle peut être très importante. Nous préférons donc utiliser le terme d'unité de lecture (Marchello-Nizia 1978) pour désigner cette "unité de base» de segmentation graphique du manuscrit médiéval. Il peut être défini de façon suivante : segment minimal de texte écrit qui réunit les conditions 
les plus favorables à l'usage de la ponctuation à ses frontières. Nous décrirons ces conditions plus précisément lorsque nous aurons défini le cadre d'analyse syntaxique que nous adoptons et que l'étude du corpus permettra de les isoler. Pour l'instant, nous nous limiterons à une description sommaire des marques de ponctuations médiévales.

Le signe de ponctuation le plus souvent utilisé dans les manuscrits en français avant le $\mathrm{XV}^{\mathrm{e}}$ siècle est le point placé à la mi-hauteur de la ligne. Il peut être suivi d'une majuscule ou d'une minuscule. A partir de la fin du XIV ${ }^{\mathrm{e}}$ siècle, dans certains manuscrits, ce rôle de " ponctuant universel » est rempli par la barre oblique. Le point et la barre oblique sont parfois utilisés dans un même manuscrit (et forment même parfois une marque « composite »), mais le plus souvent l'une de ces marques est largement dominante.

La «force » de la ponctuation n'est pas déterminée par la forme de la marque, mais par la variante de la lettre qui la suit (Lavrentiev $2000: 31, c f$. Catach $1994: 51$ ). La ponctuation faible est constituée par une marque de ponctuation suivie d'une minuscule et la ponctuation forte est déterminée par la présence d'une majuscule, qui peut être précédée d'un signe de ponctuation ou être utilisée seule. On rencontre des occurrences de la majuscule seule dans la majorité des manuscrits dès le XIII ${ }^{\mathrm{e}}$ siècle (Lavrentiev 2009 : 440). Dans certains manuscrits, la fréquence de son emploi est comparable à celle de la marque dominante, et parfois c'est la majuscule seule qui devient la marque dominante.

Certains manuscrits présentent un usage dominant soit de la ponctuation forte, soit de la faible, mais dans la majorité des cas les deux « forces » de la ponctuation sont utilisées d'une façon relativement équilibrée.

A côté de la marque dominante, tous les manuscrits ou presque présentent des occurrences de marques « occasionnelles », dont la fréquence reste souvent très basse (ne dépasse pas quelques pourcents de la totalité des occurrences des signes de ponctuation). Les échantillons restreints que nous avons utilisés sont peu adaptés à l'étude de ces marques peu fréquentes, qui jouent pourtant un certain rôle dans le système de la ponctuation. Le comma (ou punctus elevatus) qui prend la forme d'un point surmonté d'une virgule renversée ou d'une petite barre oblique, est le plus souvent suivi d'une minuscule et s'utilise parfois (jamais systématiquement) à des frontières syntaxiques importantes (entre des propositions assez longues, par exemple), mais indiquant un lien fort entre les deux unités séparées. Lors de la lecture, ce lien pouvait être signalé par une intonation montante (Marchello-Nizia 2007). Un autre usage typique du comma, celui après les interjections $h a$ et hé au début du discours direct, ne contredit pas cette hypothèse, même si l'analyse rythmique des vers montre que le comma après les interjections ne pouvait pas correspondre à une pause (Llamas Pombo 2007 : 29). La forme même de la marque semble suggérer une interprétation «mélodique » (la voix qui monte), ce qui est confirmé par certains traités latins (Hubert 1972 : 121), même si les recommandations de ces traités étaient peu suivies dans les manuscrits français.

Il existe également des manuscrits qui utilisent occasionnellement le point d'interrogation en fin d'énoncés interrogatifs ou marqués d'une forte charge émotionnelle. La forme de cette marque est parfois difficile à différencier de celle du comma. Son usage n'est jamais systématique.

D'autre marques, comme le periodus (ou punctus versus), marque ressemblant au point-virgule moderne, et destiné selon les traités de l'époque à marquer la "pause finale ", sont extrêmement rares dans les manuscrits en français à partir du XIII ${ }^{\mathrm{e}}$ siècle. Des marques « exceptionnelles », formées par exemple de plusieurs points, apparaissent parfois dans des manuscrits isolés.

Par ailleurs, un symbole appelé en France le «pied-de-mouche $»^{2}$ (en forme d'une grande lettre $c$ avec des barres verticales plus ou moins longues), le plus souvent tracé en bleu ou en rouge, peut être utilisé comme une marque de division textuelle ou de "mise en scène» des dialogues de personnages. Cette marque occupe une position transitoire entre la ponctuation «syntaxique » et la structuration globale du texte.

L'usage des marques de ponctuation est de façon générale moins dense dans les manuscrits médiévaux et dans les incunables français que dans les textes modernes, mais la variation du « taux de ponctuation » est assez importante dans les textes anciens (de 2 à 14 marques de ponctuation pour 100 occurrences-mots, comme nous l'avons déjà indiqué). La faible fréquence de la ponctuation est en partie compensée par une « abondance » de mots-connecteurs et surtout de la conjonction et (Buridant $1980: 16$; Catach 1994 : 7). 
Pour comprendre le fonctionnement de ces marques de ponctuation médiévales, il convient d'établir le cadre d'analyse linguistique qui permettra de segmenter le texte en unités clairement identifiables et indépendantes du marquage graphique avant de confronter ces unités aux pratiques de la ponctuation.

\subsection{Unité syntaxique maximale comme groupement de propositions}

En précisant les contours de son «unité phrastique», D. Creissels fait remarquer que cette notion est en effet assez proche de ce que la tradition grammaticale appelle la proposition. Mais si pour la grammaire traditionnelle la proposition est définie comme un fragment de phrase, le chercheur préfère concevoir la phrase comme un «agencement de propositions» (Creissels 1995: 39). Il suffit en effet d'étendre la notion de proposition aux "phrases simples» pour qu'elle puisse devenir une vraie unité de base d'analyse syntaxique. On pourrait ensuite dire que des propositions « syntaxiquement autonomes » sont des phrases simples et que les groupes de propositions entretenant des liens syntaxiques forts sont des phrases complexes.

Le choix de la proposition en tant qu'unité de base pour l'analyse de la ponctuation médiévale a d'ailleurs été fait par plusieurs chercheurs, à commencer par Ch. Marchello-Nizia (1978). La définition de la phrase proposée par N. Mazziotta est également très proche de celle de la proposition :

\footnotetext{
Le mot phrase renverra donc à une certaine forme d'organisation morphosyntaxique

[d'un énoncé], où le procès est exprimé par un lexème de nature verbale conjugué à un mode personnel et où le reste des constituants s'organisent autour de lui (Mazziotta 2009: 80).
}

Nous adoptons donc le principe selon lequel la proposition est une unité de base et la phrase est soit une proposition autonome, soit un ensemble de propositions reliées. Reste à définir ce qu'est la proposition et quels sont les liens entre les propositions qui constituent des phrases complexes.

En nous inspirant des travaux dans le domaine de la syntaxe sémantique (par exemple Lazard 1994) et de la description de l'« unité phrastique » de D. Creissels, nous pouvons définir la proposition comme une structure linguistique exprimant un procès (au sens très général de l'« état de choses ») et composée d'un prédicat (qui désigne le procès) et éventuellement d'un certain nombre de participants (imposés par le prédicat) et de circonstants (facultatifs).

Le prédicat est typiquement exprimé par un verbe, mais il peut être omis au cas où la juxtaposition des participants est suffisante pour exprimer le «procès », ou si le prédicat est «emprunté » à la proposition précédente.

Le cas où la juxtaposition des actants suffit à exprimer le procès est extrêmement rare en français médiéval et se limite pratiquement à des exclamations et à des proverbes, comme dans l'exemple (1) tiré de notre corpus ( $c f$. Mazziotta 2009 : 79 ; Moignet $2002: 97$ ) :

$$
\begin{aligned}
& \text { apres le sanglier le mire . et apres le cerf la biere (GastPhébChasse 619) }{ }^{3} \text { : 'Après le } \\
& \text { sanglier le médecin et après le cerf le cercueil'. }
\end{aligned}
$$

Les participants et les circonstants peuvent être exprimés par des lexèmes de nature nominale ou par des propositions, qui sont dans ce cas appelées subordonnées. Ils peuvent également être déterminés ou modifiés par des mots grammaticaux ou lexicaux, mais aussi par des propositions subordonnées.

Une proposition peut être autonome ou entretenir des liens plus ou moins forts avec d'autres propositions. Ces liens peuvent être formels (subordination, coordination, partage d'éléments) ou sémantiques. Les liens purement sémantiques sont difficiles à évaluer d'une manière objective, surtout lorsqu'il s'agit d'une langue ancienne. Seuls les critères formels seront donc retenus pour évaluer la «force» du lien entre propositions.

En cas de subordination, une proposition joue le rôle d'actant ou de circonstant, ou modifie un actant ou un circonstant d'une autre proposition. En d'autres termes, les propositions subordonnée et principale ne sont pas situées l'une à côté de l'autre, mais l'une à l'intérieur de l'autre. La relation de subordination est 
le plus souvent marquée par un connecteur subordonnant (conjonction ou pronom relatif) ou par la morphologie du verbe (mode subjonctif, forme infinitive ou participiale), même s'il existe, surtout en « très ancien » français, des subordonnées «non marquées ». La subordination est sans aucun doute un cas de lien fort entre des propositions, qui forment un ensemble syntaxique, même il existe des cas où on peut hésiter entre la subordination circonstancielle et une simple juxtaposition de propositions.

La coordination ou la juxtaposition de propositions est la situation qui pose la plus grande difficulté pour l'évaluation de la «force» du lien entre celles-ci. La simple présence d'un connecteur de coordination (comme la conjonction et) ne signifie pas que ce lien soit suffisamment fort pour former un ensemble syntaxique. Il n'est pas rare de rencontrer la conjonction et au début d'une réplique dans un dialogue ou même au début d'une division de la macrostructure textuelle marquée par une grande lettrine (Moignet 2002 : 330). Il est donc nécessaire de trouver un autre indice de la force du lien ou de poser systématiquement un «saut de phrase » à la frontière de deux propositions qui n'ont pas de relation de subordination entre elles et qui ne dépendent pas d'une même principale. C'est en effet le choix adopté par N. Mazziotta (2009: 84), ainsi que par le projet SRCMF.

Ce choix radical nous paraît toutefois contre-intuitif, car il oblige, en l'absence de subordination, à poser des limites de phrases entre deux verbes, même si ceux-ci partagent l'ensemble du schéma actanciel et le cadre spatio-temporel, comme dans l'exemple suivant :

Si le servent et honorent de quan qu'il pueent (SGraalIVQueste 77).

Dans la suite de cet article, nous proposerons un classement plus fin des liens entre des propositions coordonnées ou juxtaposées basé sur des critères de continuité thématique (qui s'exprime par la forme du sujet) et de la présence d'éléments communs à plusieurs propositions.

Un cas particulier est représenté par les incises et les incidentes. Sans marque de subordination et sans lien direct avec le prédicat de la proposition principale, celles-ci « se greffent» tout de même dans la structure de la proposition principale. On peut supposer que ces types de propositions entretiennent avec leur entourage des relations proches de la subordination, mais elles méritent de toute façon un traitement à part que nous ne pouvons pas développer ici, faute de place.

\section{Présentation du corpus}

Le corpus constitué pour le présent article comprend 29 transcriptions d'extraits (de 550 à 2000 mots environ) de manuscrits et d'imprimés français du XIII ${ }^{\mathrm{e}}$ au début du XVI siècle, soit près de 29000 mots au total. Les références des textes du corpus et les sigles utilisées dans les citations sont donnés à la fin du présent article.

Les textes du corpus représentent plusieurs domaines (littéraire, historique, didactique, etc.) et plusieurs genres textuels. Tous les textes du corpus sont en prose. Nous ne reviendrons pas ici sur les aspects méthodologiques liés à la constitution d'un corpus pour l'étude de la ponctuation médiévale, puisqu'ils ont déjà été traités dans nos publications antérieures (Lavrentiev 2007c).

\section{Classement des successions de propositions}

Deux groupes distincts se forment à l'intérieur de cette grande catégorie : les propositions relativement autonomes (dotées de tous les éléments structurels obligatoires) et les propositions qui soit partagent un élément avec une autre proposition (qui se situe habituellement dans le contexte gauche), soit sont subordonnées à une même proposition principale ou à l'un de ses éléments.

\subsection{Propositions autonomes}

Pour des raisons théoriques et en nous basant sur des observations empiriques, nous avons décidé de fonder le classement fin des propositions autonomes sur le degré de la continuité thématique exprimé par 
la forme du sujet. Nous analysons la forme du sujet dans les deux propositions qui se trouvent immédiatement à gauche et à droite de la frontière, quelle que soit la «profondeur » de leur imbrication dans des constructions subordonnées éventuelles.

\subsection{1 « Nouveau » sujet nominal}

La plus grande autonomie concerne les propositions avec un sujet nominal, dont le référent n'est pas présent sous une forme ou une autre dans la proposition qui la précède immédiatement. C'est une frontière assez proche de la limite de phrase dans la perception du lecteur moderne. Plus de la moitié des occurrences (255 sur 498) de cette frontière portent une ponctuation forte (point, point d'interrogation ou point d'exclamation) dans les éditions modernes des textes de notre corpus, sans compter les 75 occurrences de ponctuation moyenne (point-virgule ou deux-points) et les 139 occurrences de la ponctuation faible (virgule). Autrement dit, près de 95\% (469 sur 498) des occurrences de ce type de frontières entre propositions sont ponctuées dans les éditions modernes. Dans les manuscrits cette frontière est également très «favorable » à la présence d'une ponctuation, mais des exceptions existent. Dans l'exemple suivant, la frontière de ce type n'est pas ponctuée dans le manuscrit, mais l'éditeur (Micha 1979) a choisi d'y mettre une ponctuation forte :

$$
\begin{aligned}
& \text { et quant } i l \text { le uoient si l'acolent et besent }[>.]^{4} \text { et lors comence la ioie que li cosin } \\
& \text { firent li .i. de l'autre. (SGraalIVQueste 1119). }
\end{aligned}
$$

Dans certains cas, les éditeurs ont jugé que le lien entre les propositions correspondantes était suffisamment fort pour n'utiliser qu'une ponctuation faible, voire ne mettre aucune ponctuation :

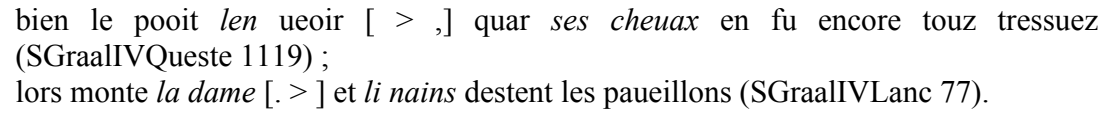

\subsubsection{Changement de rôle}

Cette catégorie concerne les propositions où le référent du sujet nominal (6), pronominal (7) ou nul (8) de la proposition qui suit la frontière est présent dans la proposition précédente :

(7) ne ele n'est moie [ $>$, ] ne ie n'auroie le corage de metre i main (SGraalIVQueste 77) ;

(8) et il monte et la damoiselle ausint [ $>$.] si se partent de laienz sanz autre congie (SGraalIVQueste 1119).

Dans les éditions modernes, les frontières de ce type portent une ponctuation forte dans un tiers des cas environ (63 occurrences sur 191).

\subsubsection{Reprise de sujet}

Il s'agit de successions de propositions où le sujet pronominal personnel de la deuxième proposition renvoie au même référent que celui de la proposition précédente :

Si met la main a l'espee et tret [. > ,] mes il ne la puet auoir (SGraalIVQueste 77).

Sous certaines conditions précises, sont classés dans cette catégorie les cas où le sujet de la deuxième proposition n'est pas exprimé. Nous reviendrons sur ces cas dans la section 4.2.1.

Les éditeurs scientifiques placent une ponctuation forte dans près de $40 \%$ des occurrences de ce type de frontière (100 occurrences sur 258). 


\subsubsection{Propositions impersonnelles}

Les propositions impersonnelles (dont le sujet peut être ou ne pas être exprimé) constituent une catégorie à part :

et li rois comande que les tables soient mises [. > ,] car il est tens de mengier (SGraalIVQueste 77);

n'i metrai ia la main [. > ,] car ce seroit folie se ie tendoie a auoir la (SGraalIVQueste 77).

Ce type de frontière est nettement moins fréquent que les trois précédents (68 occurrences seulement dans notre corpus). La ponctuation forte y est utilisée par les éditeurs dans près d'un tiers des cas.

\subsection{Propositions ayant des éléments communs}

\subsubsection{Sujet commun}

Il s'agit d'une frontière entre deux propositions, dont la deuxième n'a pas de sujet exprimé et où tout (la forme du verbe et le sens) porte à croire que le sujet est le même que dans la proposition précédente.

Puisque l'expression du sujet en français médiéval n'est pas obligatoire, on peut se poser la question de savoir s'il existe une réelle différence entre cette catégorie et celle présentée dans la section 4.1.3 cidessus (où le sujet, qui renvoie toujours au même référent, est exprimé par un pronom). Prenons l'exemple suivant, où nous avons indiqué les frontières entre les propositions par des chiffres en indice devant les crochets :

$$
\begin{aligned}
& \text { D'ileuques [Homar] se traist uers damas }{ }_{1}[>] \text { et assist la cite }{ }_{2}[>] \text { et assailli }{ }_{3}[.>\text {, ] et } \\
& \text { la prist par force }{ }_{4}[.>\text {. ] Car il auoit si grant plante de gent . (GuillTyr } 828 \text { ). }
\end{aligned}
$$

L'intuition, appuyée par la ponctuation scribale, nous dit que la division à la quatrième frontière est plus forte que les précédentes. La présence du pronom sujet et du connecteur car, qui à la différence de et ne s'utilise qu'au niveau interpropositionnel, ainsi que le changement du temps verbal sont des marques segmentales de cette autonomie. On peut également apercevoir que le lien entre les propositions assist la cite et assailli est plus fort qu'entre les autres, car celles-ci partagent l'ensemble du schéma actanciel (l'objet la cite, en plus du sujet) et les événements qu'elles décrivent sont simultanés ou presque.

Les facteurs qui peuvent intervenir dans l'appréciation de la proximité entre deux propositions « avec un sujet commun» sont multiples. Pour assurer une procédure d'analyse stable et efficace, nous avons élaboré l'algorithme suivant :

Nous considérons qu'il s'agit d'une proposition ayant « son propre » sujet non exprimé :

- si le cadre spatio-temporel de la deuxième proposition est différent de celui de la première (ce qui est signalé par des adverbes, des circonstants exprimés par des syntagmes nominaux ou par des propositions subordonnées) : exemple (13);

- $\quad$ si les connecteurs car ou si sont utilisés : exemples $\left(12_{4}\right),(14)$ et $\left(15_{2}\right)$;

- si la conjonction et reliant les deux propositions est suivie d'un adverbe à valeur temporelle, causale, finale ou concessive (lors, donc, pour ce, neporquant, etc.) : exemple (12 1 ).

(13) Et l'apostoles manda en france au saint home . que il preechast des crois par s'auctorite [. > ;] Et apres i enuoia .i. sien cardonnal (Villeh 12203);

(14) il regarde l'enfant [ $>$,] si le uoit garniz de totes biautez (SGraalIVQueste 1119);

(15) si uoit gesir ses .ii. cosins bohort et lionel en .ii. $\operatorname{liz}_{1}[>$.] et lors est a merueilles liez ${ }_{2}[>$,] si les esueille (SGraalIVQueste 1119);

En revanche, il s'agit d'un seul sujet « partagé » par les deux propositions :

- $\quad$ si les deux propositions partagent l'ensemble du schéma actanciel : exemple (2);

- si les prédicats des deux propositions n'ont pas d'autres arguments que le sujet : exemple (16); 
- si le prédicat de la deuxième proposition est «nu» et peut être interprété soit comme partageant l'objet de la première, soit comme un emploi absolu : exemple (17);

- les deux propositions ont (en plus du sujet) des participants, des circonstants ou des subordonnées en commun : exemple (18) ;

- les deux prédicats ont un ou plusieurs arguments différents, mais aucun indice suffisant pour supposer qu'il s'agit d'un sujet « non exprimé » (19).

(16) la terre tremble $[>$, ] crole $[>$,] et fent . (ImMonde 1495);

(17) [Messire Gauuain] prent l'espee par le heut [ $>$ ] et sache (SGraalIVQueste 77);

(18) et + por+ce m'en tendrai ie [ $>$, ] ne n'i metrai ia la main (SGraalIVQueste 77);

(19) Et il monte [ $>$ ] et se part de la cort (SGraalIVQueste 77).

Les critères précis choisis pour décider s'il s'agit ou non d'un « sujet commun » peuvent se discuter, mais dans la majorité des cas ils permettent d'obtenir des résultats qui s'accordent avec les tendances de la ponctuation dans les sources primaires (manuscrits et incunables) aussi bien que dans les éditions modernes. Ainsi, dans les sources primaires, les frontières entre les propositions avec la « reprise du sujet » sont ponctuées dans $75 \%$ des cas (ponctuations forte et faible confondues) ${ }^{5}$, tandis que ce taux tombe à $36 \%$ en cas de « sujet commun ». Dans les éditions, le pourcentage des frontières ponctuées est plus élevé dans les deux cas, mais la différence entre les catégories est aussi bien marquée (90\% des frontières ponctuées contre $48 \%$ respectivement).

\subsubsection{Subordonnées coordonnées}

Nous classons les successions de propositions dans cette catégorie uniquement s'il n'y a aucun doute sur le fait que les deux propositions à gauche et à droite de la frontière sont subordonnées à une même principale, par exemple en cas de renouvèlement du marqueur de subordination (20) ou si les deux subordonnées précèdent la principale avec la coordination (conjonction et) et la réexpression du sujet par le pronom $(i l)$, ou s'insèrent dans une structure plus grande $(21)$.

$$
\begin{aligned}
& \text { cil de la sale uirent seoir le cheualier ou siege que tant preudome auoient redoute [. > ] } \\
& \text { et ou tantes granz auentures estoient auenues par maintes foiz (SGraalIVQueste 77); } \\
& \text { Qvant li rois fu uenu el mostier [. > ] et il uit que lancelot fu uenuz [ > ,] et il ot amene } \\
& \text { boort et lion si lor fet mout grant ioie (SGraalIVQueste 77). }
\end{aligned}
$$

\subsection{3 « Prédicat commun » (ellipse du verbe)}

Cette catégorie concerne les cas où la deuxième proposition ne contient pas de verbe conjugué (ellipse du verbe, selon la grammaire traditionnelle) :

$$
\begin{aligned}
& \text { Et desdictes liquides les vnes sont consonans [ }>\text {, ] les autres demi voyeux [ }>\text { ] et les } \\
& \text { autres mutes (Desch } 840 \text { ); } \\
& \text { Et li .ii. mistrent les cierges sus la table }{ }_{1}[.>\text {,] et li tierz la toaille lez le saint vessel } \\
& \text { }[.>\text {, et li quarz tint la lance tote droite sus le saint vessel (SGraallVQueste } 77 \text { ); } \\
& \text { Et venismes au giste cedict soir a plaisance qui est grande et belle cite [ }>\text {.] De } \\
& \text { plaisance a cremonna qui est cite grande et belle }[/>\text {.] D'illec a peticolle }[>,] \text { apres a } \\
& \text { wastala au pont d'ueil (Anglure 15217). }
\end{aligned}
$$

Dans (22), nous avons une simple omission de la copule, ce qui est le cas le plus fréquent. Dans $\left(23_{1}\right)$, c'est le verbe 'mettre' qui est omis, tandis que tous les participants sont différents dans les deux propositions. Notons que dans la suite de cet extrait $\left(23_{2}\right)$, nous avons une succession de type «nouveau sujet », car il s'agit d'une proposition autonome dotée d'un verbe et d'un sujet nominal (adjectif ordinal substantivé). Dans (24), qui est un exemple tiré d'un récit de voyage, nous avons toute une série de séquences composées de syntagmes prépositionnels indiquant le point de départ et d'arrivée d'un déplacement (ou juste le point d'arrivée) éventuellement complétées par des relatives. Nous considérons chacun de ces groupes comme une proposition elliptique, puisqu'il s'agit à chaque fois d'un événement indépendant localisé dans le temps et dans l'espace. 


\subsubsection{Sujet différent, objet ou complément commun}

Notre classement prévoit une catégorie, théoriquement possible, où des propositions ayant des sujets différents partagent un même objet ou un autre complément essentiel :

*Les journalistes écrivent et le public lit les articles.

Notre corpus ne présente toutefois aucune occurrence de ce type de construction. Par ailleurs, dans des constructions de ce type, il serait toujours possible d' " accrocher » l'objet uniquement au verbe le plus proche et de considérer qu'il s'agit, pour l'autre verbe, d'un emploi absolu.

Comme c'était le cas des propositions «autonomes», les différents types de propositions ayant des éléments communs présentent, d'après les résultats de l'analyse de notre corpus, plus de traits communs que de différences.

\section{Conclusion}

A l'issue de l'analyse de l'emploi de la ponctuation dans les différents types de succession de propositions dans un corpus de transcriptions de manuscrits, d'incunables et de leurs éditions scientifiques, nous pouvons formuler l'hypothèse selon laquelle l'« unité syntaxique maximale » (ou la « phrase ») peut être définie comme un agencement de propositions qui, soit entretiennent des relations de subordination (dans ce cas, il s'agit de l'enchâssement d'une proposition dans une autre), soit « partagent » un ou plusieurs éléments constitutifs (prédicat, sujet, objet ou complément). Cette hypothèse est confirmée par l'analyse factorielle de la distribution des différents types de ponctuation (forte, faible ou absente) selon les types de «frontières ponctuables ». Le traitement des frontières de propositions ayant des éléments communs est en effet similaire à celui des frontières entre les propositions subordonnées et principales, tandis que les frontières des propositions «autonomes » présentent une distribution totalement différente sur l'ensemble du corpus.

Des unités autonomes, que l'on pourrait nommer «phrases », existent donc bien en français médiéval, auxquelles les critères proposés par la grammaire pour leur définition ne sont pas directement applicables, mais pour lesquelles des critères de reconnaissance sont possibles. Une étude plus fine des types d'agencement de propositions et de la ponctuation qui les accompagne sur un corpus de transcriptions de sources primaires plus grand et plus diversifié que le nôtre permettrait sans doute de préciser davantage les contours de cette unité.

\section{Corpus étudié}

Anglure 15217 - Ms. Paris, BnF, fr. 15217 (2e m. XV s.), fo27r-30v: Ogier d'Anglure, Le Saint voyage de Jherusalem.

BeaumCout 11652 - Ms. Paris, BnF, fr. 11652, (ca. 1300), fo 4r-5r, Philippe de Beaumanoir, Coutumes de Beauvaisis.

Chartier 126 - Ms. Paris, BnF, fr. $126\left(1^{\mathrm{ère}} \mathrm{m} . \mathrm{XV}^{\mathrm{e}}\right.$ s.), 191r-192v : Alain Chartier, Quadrilogue invectif.

ComteArt 11610 - Ms. Paris, BNF, fr. 11610 (entre 1453 et 1467), fo 1r-4r, Roman du comte d'Artois.

Desch 840 - Ms. Paris, BnF, fr. 840 (ca. 1400), 394r-396v : Eustache Deschamps, Art de dictier.

Dolet 1540 - Imprimé à Lyon, Estienne Dolet, 1540 (BnF, Gallica), p. 12-14 : Estienne Dolet, La maniere de bien tradvire d'une langue en aultre.

Egipte 769 - Ms. Lyon, Bibliothèque municipale, 769 (XV's.), f $4 \mathrm{v}-5 \mathrm{r}$ : Fragment d'une description de l'Orient.

FroissChron3 869 - Ms. Vatican, Reg. Lat. 869 (entre 1399 et 1405), fo 1v - 2r : Jean Froissart, Chroniques (livre premier).

GastPhébChasse 619 - Ms. Paris , BnF, fr. 619 (vers 1440), f 1r-4v : Gaston Phébus, Livre de chasse. 
GastPhébChasse 765 - Ms. Bibliothèque municipale de Lyon, $765\left(\mathrm{XV}^{\mathrm{e}} \mathrm{s}\right.$.), f $\mathrm{f}^{\mathrm{o}}$ 100r et 102v : Gaston Phébus, Livre de chasse.

GuillTyr 828 - Ms. Lyon, Bibliothèque municipale, 828 (XIV s.), fo $1 \mathrm{r}$ et $5 \mathrm{r}$ : Guillaume de Tyr, Le liures deracles.

ImMonde 1495 - Imprimé à Paris, Jehan Treperl, entre 1492 et 1498 (BnF rés. D. 4722), fo 2r-2v : Gossouin, Image du monde (en prose).

ImMonde 1522 - Imprimé à Paris, Jehan Janot, ca. 1522, p. 8-9 : Gossouin, Image du monde (en prose).

ImMonde 574 - Ms. BnF, fr. 574 (ca. 1315-1320), fo 4v-6r : Gossouin, Image du monde (en prose).

ImMonde 587 - Ms. Paris, Sainte-Geneviève, 587; (fin XIII / début XIV ${ }^{\mathrm{e}}$ s.), f ${ }^{\circ} 172 \mathrm{v}$ : Gossouin, Image du monde (en prose).

JGerson 13318 - Ms. Paris, BnF, fr. 13318 (XV s.), fo 165v-170r : Jean Gerson, Sermon pour la fête de la Sainte Trinité.

Joachim 768 - Ms. Lyon, Bibliothèque municipale, 768 (XIV s.), f 97v-98r : La prophecie de Mestre Joachin l'astronomien.

Joinv 13568 - Ms. Paris, BnF, fr. 13568 (ca 1335), p. 1-7 : Jean de Joinville, Vie de saint Louis.

Monstre 2682 - Ms. Paris, BnF, fr. 2682, (XV s.), fo 233r-233v : Enguerrand de Monstrelet, Chroniques (livre 2).

MortArtu 1488 - Imprimé à Paris, Jehan du Pré, 1488 (BnF, rés. Y² 47), fo 366r : La mort au roy Artus.

QJoyes 1052 - Ms. Rouen, Bibliothèque municipale, 1052 (daté 1464), fo 87r-89r : Les .xv. joies de mariage.

Serments 9768 - Ms. Paris, BnF, lat. 9768 (ca. 1000), fo 13r : Les serments de Strasbourg (Chronique de Nithard).

SGraalIVLanc 77 - Ms. Lyon, Bibliothèque municipale, fonds du Palais des arts 77 (XIII ${ }^{\mathrm{e}}$ s.), fo 8v-9r : Lancelot du lac.

SGraalIVQueste 1119 - Ms. Paris, BnF, n.a. fr. 1119 (XIII ${ }^{\mathrm{e}}$ - début XIV ${ }^{\mathrm{e}}$ s.), fo 138r-141v : Queste del saint graal.

SGraalIVQueste 77 - Ms. Lyon, Bibliothèque municipale, fonds du Palais des arts 77 (XIII ${ }^{\mathrm{e}} \mathrm{s}$ ), $\mathrm{f}^{\mathrm{o}} 160 \mathrm{v}-164 \mathrm{r}$ : Queste del saint graal.

SimPhares 1357 - Ms. Paris, BnF, fr. 1357 (1494-1498), fo 1v-5r: Simon de Phares, Recueil des plus celebres astrologues.

Ursins 2701 - Ms. Paris, BnF, fr. 2701 (3 $3^{\mathrm{e}}$ q. XV s.), fo 121r-121v : Jean Juvenal des Ursins, Proposition...

Villeh 12203 - Paris, BnF, ms. fr. 12203 (XIII ${ }^{\mathrm{e}}$ s.), fo 69r-70v: Geoffroi de Villehardouin, Conqueste de constantinople.

Villeh 587 - Ms. Oxford, Bodleian, Laud. Misc. (XIV s.), fo 69r-70v : Villehardouin, Conqueste de constantinople.

\section{Références bibliographiques}

Baddeley, S. (2001). La ponctuation de manuscrits français du $\mathrm{IX}^{\mathrm{e}}$ au XII ${ }^{\mathrm{e}}$ siècle. Liaisons HESO/AIROE, 32-33, 139-149.

Barbance, C. (1995). La ponctuation médiévale : quelques remarques sur cinq manuscrits du début du XV siècle. Romania, 113, 455-456, 505-525.

Buridant, C. (1980). Le strument et et ses rapports avec la ponctuation dans quelques textes médiévaux. In DessauxBerthonneau, A. (éd.), Théories linguistiques et traditions grammaticales, Villeneuve-d'Asq: Presses Universitaires de Lille, 13-53.

Buridant, C. (2007). Grammaire nouvelle de l'ancien français. Paris : SEDES.

Careri, M., Fery-Hue, F., Gasparri, F., Hasenohr, G., Labory, G., Lefèvre, S., Leurquin, A. et Ruby, C. (2001). Album de manuscrits français du XIII siècle. Mise en page et mise en texte. Rome : Viella.

Catach, N. (1994). La ponctuation. Paris : Presses universitaires de France.

Chomsky, N. (1986). La nouvelle syntaxe. Paris : Éditions du Seuil. 
Combettes, B. (2000). La ponctuation et l'énoncé complexe au XVI ${ }^{\mathrm{e}}$ siècle (l'usage des deux points chez Jean de Léry). La Licorne, 52, 63-79.

Creissels, D. (1995). Éléments de syntaxe générale. Paris : Presses universitaires de France.

Creissels, D. (2006). Syntaxe générale une introduction typologique. Volume 2. La phrase. Paris : Hermes Science.

Grevisse, M. et Goosse, A. (2007). Le bon usage : grammaire française. Bruxelles ; Louvain-la-Neuve : Duculot ; de Boeck.

Hubert, M. (1972). Le vocabulaire de la "ponctuation" aux temps médiévaux : Un cas d'incertitude lexicale. Bulletin du Cange, Archivum latinitatis Medii Avi, 38, 57-167.

Lavrentiev, A. (2000). A propos de la ponctuation dans l'Image du monde. La Licorne, 52, 23-37.

Lavrentiev, A. (2007a). Pour une méthodologie d'étude de la ponctuation médiévale basée sur une approche typologique. In Combettes, B. et Marchello-Nizia, C. (éds.), Études sur le changement linguistique en français, communications du Colloque "Diachro 2 ", (Actes de colloque, Paris, 15-17 janvier 2004), Nancy : Presses Universitaires de Nancy, 191-204.

Lavrentiev, A. (2007b). Connecteurs et ponctuation comme outils de structuration du texte à travers les manuscrits et incunables français en prose du XIII ${ }^{\mathrm{e}}$ au début du XVI ${ }^{\mathrm{e}}$ siècle. In Vanderheyden, A., Mortelmans, J., de Mudler, W. et Venckeleer, Th. (éds.), Texte et discours en Moyen français, (Actes du XI ${ }^{\mathrm{e}}$ Colloque international sur le moyen français Anvers, 19-21 mai 2005), Turnhout : Brepols, 149-162.

Lavrentiev, A. (2007c). Typologie textuelle pour l'étude linguistique de manuscrits français médiévaux ». In: Lavrentiev, A. (dir.), Systèmes graphiques de manuscrits médiévaux et incunables français: ponctuation, segmentation, graphies, (Actes de journée d'études, Lyon, 6 juin 2005), Chambéry : Université de Savoie, 49-66.

Lavrentiev, A. (2009). Tendances de la ponctuation dans les manuscrits et incunables français en prose, du XIII au $X V^{e}$ siècle. Thèse de doctorat en sciences du langage, Lyon : ENS LSH.

Lazard, G. (1994). L'actance. Paris : Presses universitaires de France.

Llamas Pombo, E. (2001). La ponctuation du vers dans un manuscrit du XIV ${ }^{\mathrm{e}}$ siècle. Liaisons HESO/AIROE, 32-33, 151-171.

Llamas Pombo, E. (2007). Réflexions méthodologiques pour l'étude de la ponctuation médiévale. In Lavrentiev, A. (éd.), Systèmes graphiques de manuscrits médiévaux et incunables français: ponctuation, segmentation, graphies, Chambéry : Université de Savoie, 11-48.

Llamas Pombo, E. (2008). Ponctuer, éditer, lire. État des études sur la ponctuation dans le livre manuscrit. Syntagma. Revista del Instituto de Historia del libro y de la lectura, 2, 129-171.

Marchello-Nizia, C. (1978). Ponctuation et "unités de lecture" dans les manuscrits médiévaux ou : je ponctue, tu lis, il théorise. Langue française, 40, 32-44.

Marchello-Nizia, C. (1979). La notion de "phrase" dans la grammaire. Langue française, 41, 35-48.

Marchello-Nizia, C. (2007). Le comma dans un manuscrit en prose du $13^{\mathrm{e}}$ siècle : grammaticalisation d'un marqueur de corrélation, ou marquage d'intonation ? In Bertrand, O. et al. (éds.), Discours, diachronie, stylistique du français. Études en hommage à Bernard Combettes, Berne : Peter Lang, 293-305.

Mazziotta, N. (2008). Ponctuation et syntaxe en ancien français : Étude d'un corpus de chartes écrites à Liège avant 1292. Méthode de travail. In Durand, J., Habert, B. et Lacks, B. (éds.), Congrès mondial de linguistique française - $C M L F^{\prime} 08$, (Actes de colloque, Paris, 9 - 12 juillet 2008), Paris : Institut de linguistique française, 267-277.

Mazziotta, N. (2009). Ponctuation et syntaxe dans la langue française médiévale : Étude d'un corpus de chartes originales écrites à Liège entre 1236 et 1291. Tübingen : Max Niemeyer Verlag.

Mel'čuk, I. A. (1988). Dependency syntax theory and practice. Albany : State University of New York Press.

Micha, A. (éd.) (1979). Lancelot (D'une aventure d'Agravain jusqu'à la fin de la quête de Lancelot par Gauvain et ses compagnons). Genève ; Paris : Droz.

Moignet, G. (2002). Grammaire de l'ancien français. Paris : Klincksieck.

Mortara Garavelli, B. (éd.) (2008). Storia della punteggiatura in Europa, Roma : Laterza. 
Nunberg, G. (1990). The linguistics of punctuation. Stanford, CA: Center for the Study of Language and Information.

Parkes, M. B. (1992). Pause and effect: an introduction to the history of punctuation in the West. Aldershot: Scolar Press.

Pignatelli, C. (2007). Présence et fréquence de la ponctuation dans les manuscrits en vers du XIII ${ }^{\mathrm{e}}$ siècle : les huit manuscrits du Chevalier de la Charrette au banc d'essai. In Lavrentiev, A. (éd.), Systèmes graphiques de manuscrits médiévaux et incunables français : ponctuation, segmentation, graphies, Chambéry: Université de Savoie, 85-105.

Stiennon, J. (1991). Paléographie du Moyen Âge. Paris : Armand Colin.

Tesnière, L. (1965). Éléments de syntaxe structurale. Paris : Klincksieck.

${ }^{1}$ Syntactic reference Corpus for Medieval French. Projet co-financé par l'Agence nationale de la recherche (ANR, France) et par la Deutsche Forschungsgemeinschaft (DFG, Allemagne), dirigé par Sophie Prévost (UMR Lattice) et par Achim Stein (Université de Stuttgart).

${ }^{2}$ Le terme de pied-de-mouche est couramment utilisé pour désigner la marque en question dans les recherches consacrées à la ponctuation française médiévale (par exemple, Barbance 1995: 509) et dans les albums paléographiques Maria Careri, Françoise Fery-Hue, Françoise Gasparri, et al. 2001. Il est par ailleurs mentionné dans Le bon usage de M. Grevisse Maurice Grevisse and André Goosse. 2007. J. Stiennon Jacques Stiennon. 1991 lui donne pourtant un autre sens : «petit trait de couleur surchargeant la première lettre d'un mot pour la mettre en valeur ». M. Parkes (1992) et les auteurs de la récente Storia della punteggiatura in Europa (Mortara Garavelli éd. 2008) utilisent, pour la marque en forme de 〈 $\mathbf{C}\rangle$, le terme de paraph (ou paraffo).

${ }^{3}$ Les références des citations du corpus sont composées du sigle bibliographique du DEAF (http://www.deafpage.de/) s'il existe pour le texte en question et de la cote du manuscrit ou de l'année d'impression pour les incunables. La liste complète des références est présentée à la fin du présent article.

${ }^{4}$ Nous utiliserons les crochets et le chevron comme dans cet exemple pour indiquer à la fois la ponctuation du manuscrit (à gauche du chevron) et celle de l'édition (à droite du chevron). Les sujets des deux propositions adjacentes sont mis en italiques.

${ }^{5} \mathrm{La}$ fréquence relative des ponctuations fortes et faibles varie considérablement selon les sources, mais dans tous les cas il s'agit d'une position favorable à la ponctuation forte. 